\title{
Improving Animation Technology through Digital Transformation
}

\author{
Lincoln Wallen \\ Chief Technology Officer \\ DreamWorks Animation \\ 1000 Flower Street \\ Glendale \\ California \\ USA \\ www.dreamworksanimation.com \\ lincoln.wallen@dreamworks.com
}

\section{ABSTRACT}

DreamWorks Animation creates high-quality family entertainment centred on groundbreaking computer generated (CG) animated feature films, which include the properties of Shrek, Kung Fu Panda, and How to Train Your Dragon. Five years ago,
DreamWorks Animation completed the single most significant business transformation in the studio's 20 year history. Today, Lincoln Wallen (CTO of DreamWorks Animation) is sharing how the studio is optimising their digital transformation to achieve bottom line results. 